\title{
Larvicidal Activity of Sterol Carrier Protein-2 Inhibitor in Four Species of Mosquitoes
}

\author{
Ryan T. Larson, Vilena Wessely, Zhisheng Jiang, and Que Lan ${ }^{1}$ \\ Department of Entomology, University of Wisconsin-Madison, Madison, WI 53706
}

\begin{abstract}
A previous report has shown that mosquito sterol carrier protein-2 inhibitors (SCPIs) are larvicidal to larvae of the yellowfever mosquito, Aedes aegypti (L.) (J. Lipid Res. 46: 650-657, 2005). In the current study, we tested SCPI-1 in an additional four mosquito species for larvicidal activities: Culex pipiens pipiens, Anopheles gambiae, Culex restuans, and Aedes vexans. Cholesterol accumulation in SCPI-treated Ae. aegypti fourth instars was examined. SCPI-1 is lethal to all tested mosquito species, with the $\mathrm{LC}_{50}$ value ranging from 5.2 to $15 \mu \mathrm{M}$ when treatments started at the first to third instar. However, $\mathrm{LC}_{50}$ values increase to from 5.2 to $38.7 \mu \mathrm{M}$ in treatments started at first and fourth instar, respectively. The results indicate that the lethal effect of SCPI-1 decreases with the growth of larvae, which suggests that SCPI-1 is more effective before the larvae reach final growth period (the last instar). SCPI-1 suppressed cholesterol uptake in Ae. aegypti fourth instars, suggesting that one of the modes of action of SCPI-1 is via reduction in cholesterol absorption.
\end{abstract}

\section{Keywords}

cholesterol; insecticide; larvicide; Aedes; sterol carrier protein

\begin{abstract}
Yellowfever mosquito, Aedes aegypti (L.), transmits yellow fever and dengue hemorrhagic fever (DHF). A potential population of 2 billion people living in tropical and subtropical regions is at risk for these mosquito-borne viral diseases (Halstead 1992, CDC 2003). One of the effective approaches in preventing the transmission of yellow fever and DHF is mosquito population control by using traditional insecticides (Focks et al. 2000). However, this has been compromised by the development of insecticide resistance (Mourya et al. 1993, Mourya et al. 1994, Rawlins and Wan 1995, Rawlins 1998). Cholesterol uptake/transport has been used as a potential target for the development of new mosquito larvicides (Kim et al. 2005).

Insects lack key enzymes in the cholesterol synthesis pathway (Zdobnov et al. 2002). Insect dependence on dietary cholesterol highlights the critical physiological process of cholesterol absorption and translocation, without which insects cannot grow and reproduce. Cholesterol is highly hydrophobic (Renshaw et al. 1983). Carrier proteins mediate the delivery of cholesterol by shielding the hydrophobic moiety of cholesterol from the aqueous environment of the cytoplasm or hemolymph (Chino and Kitazawa 1981, Yancey et al. 1996, Arrese et al. 2001, Schroeder et al. 2001). A sterol carrier protein (SCP) is needed to transport cholesterol intracellularly from the lumenal side to the basal side of the midgut epithelium or from lipid droplet to the cytoplasmic membrane in the fat body in insects. Moreover, studies have shown that Aedes SCP-2, an intracellular sterol carrier protein, is at least partially responsible for cellular cholesterol transfer in mosquitoes (Lan and Massey 2004 and Blitzer et al. 2005).
\end{abstract}


SCP-2 belongs to a family of proteins containing a sterol-binding domain (SCP-2 domain). The vertebrate SCP-2 has a peroxisome localization sequence in the $\mathrm{C}$ terminus, targeting these proteins to the peroxisome (Moller et al. 1999, Gallegos et al. 2001). However, the mosquito AeSCP-2 seems to be a unique nonperoxisomal and low-molecular-weight protein in the SCP-2 gene family (Krebs and Lan 2003, Lan and Massey 2004). The AeSCP-2 homolog is found in Anopheles gambiae genome with $>85 \%$ similarity (Lan and Wessely 2004, Vyazunova et al. 2007), indicating a highly conserved nature of mosquito SCP-2 proteins between species.

The substantial divergences in the structure and cellular localization of SCP-2s (Dyer et al. 2003, Lan and Massey 2004) suggest that there may be functional differences between the vertebrate and mosquito SCP-2s. AeSCP-2 inhibitors (SCPIs) have been identified from a 16,000-compound chemical library (Kim et al. 2005). SCPIs suppress cholesterol uptake by $30 \%$ at $1 \mu \mathrm{M}$ concentrations (Kim et al. 2005), consistent with the finding that AeSCP-2 is partially responsible for cellular cholesterol incorporation (Lan and Massey 2004). The result is also consistent with the effects of AeSCP-2 silencing on cholesterol uptake in vivo (Blitzer et al. 2005). In this study, we tested SCPI-1 in several mosquito species to investigate the larvicidal spectrum of SCPIs. The effects of SCPIs on cholesterol uptake were examined in Ae. aegypti larvae in vivo.

\section{Materials and Methods}

\section{Chemicals}

Chemicals and reagents were purchased from Sigma-Aldrich (St. Louis, MO), Fisher Scientific (Hampton, NH), and MP Biomedicals (Irvine, CA) if their origins were not mentioned in the text. $\left[1,2-{ }_{-}^{3} \mathrm{H}(\mathrm{N})\right]$ Cholesterol $(40 \mathrm{Ci} / \mathrm{mmol})$ was purchased from the American Radiolabeled Chemicals (St. Louis, MO). AeSCP-2 inhibitors SCPI-1 [N-(4-\{[4-(3,4-dichlorophenyl)-1,3thiazol-2-yl]amino \}-phenyl)acetamidehydrobromide] and SCPI-2 [8-chloro-2-(3methoxyphenyl)-4,4-dimethyl-4,5-dihydroisothiazolo[5,4-c]quinoline-1(2H)-thione] were purchased from ChemBridge Corporation (San Diego, CA), with at least 90\% purity.

\section{Mosquitoes}

Ae. aegypti were taken from an inbred laboratory strain (Rockefeller). Larvae were reared at $26^{\circ} \mathrm{C}$ in $70-80 \%$ humidity at a photoperiod of $14: 10$ (L:D) h. Larvae hatching during a 25min period were collected and used in experiments, and the density was $\approx 1,000$ larvae per $2,000 \mathrm{ml}$ of double-distilled water. Food was prepared by adding $5 \mathrm{ml}$ of distilled water into $500 \mathrm{mg}$ of dry fish food (TretraMin, Tetra Holding, Inc., Blacksburg, VA). The larvae were fed 10 drops of food/1,000 ml double-distilled water every day except for the first $2 \mathrm{~d}$ when they were fed only once. Adults were maintained using $10 \%$ glucose and defibrinated rabbit blood (Lampire Biological Laboratories, Pipersville, PA) in $70-80 \%$ humidity at $26^{\circ} \mathrm{C}$.

An. gambiae were taken from an inbred laboratory strain (G3). Newly hatched larvae were reared at $26^{\circ} \mathrm{C}$ in $70-80 \%$ humidity at a photoperiod of $14: 10$ (L:D) $\mathrm{h}$ and were fed with 1:10 diluted food (100 g of Vita-Pro Plus fish power staple fish food (M. Reed Enterprises, Sutter Creek, CA) and $50 \mathrm{~g}$ of brewer's yeast (Fisher Scientific) dissolved in $450 \mathrm{ml}$ of double-distilled $\mathrm{H}_{2} \mathrm{O}$.

Cx. pipiens were taken from an inbred laboratory strain Cx. pipiens pipiens. Eggs were hatched overnight in distilled water, and first instars were used in the toxicity assays. Larvae were reared at $26^{\circ} \mathrm{C}$ in $70-80 \%$ humidity at a photoperiod of 14:10 (L:D) h, with fish food (TetraMin).

Cx. restuans larvae were taken from the wild. The location of Culex breeding sites was obtained from the Madison-Dane County Department of Public Health West Nile virus database. Mosquito larvae were collected between June and August 2006 using a $350 \mathrm{ml}$ dipper. After 
being collected, the mosquitoes were placed in a plastic container, which was then placed in an ice-filled cooler. At least 20 larvae were obtained from each sample for identification. The larvae were killed using 70\% ethanol before they were identified with Mosquitoes of Indiana Key (Siverly 1972). Only samples containing $\geq 90 \%$ larvae from a single species were used in toxicity assays. In most cases, the larvae were between second and third stadium.

Locations for larvae collections were as follows: $43^{\circ} 05^{\prime} 15.09^{\prime \prime} \mathrm{N}, 89^{\circ} 30^{\prime} 48.18^{\prime \prime} \mathrm{W}, 22-\mathrm{VI}-/$ 2006, 60; all Cx. restuans; $43^{\circ} 07^{\prime} 04.94^{\prime \prime} \mathrm{N}, 89^{\circ} 18^{\prime} 54.05^{\prime \prime} \mathrm{W}, 5-\mathrm{VII}-2006$; 55 Cx. restuans, 5 Cx. pipiens; and $43^{\circ} 02^{\prime} 24.60^{\prime \prime} \mathrm{N}, 89^{\circ} 27^{\prime} 28.98^{\prime \prime} \mathrm{W}$, VII-17-2006, 18 Cx. restuans, Ae. vexans; 1 Cx. pipiens.

\section{Toxicity Assays}

Larvae of Ae. aegypti, Cx. pipiens pipiens, and An. gambiae were treated separately with increasing concentrations of AeSCP-2 inhibitor-1 (SCPI-1). SCPI-1 was diluted from a stock solution (100 $\mathrm{mM}$ in dimethyl sulfoxide [DMSO]) to various concentrations in double-distilled water. Third instars of An. gambiae (7-8 d old) and Cx. Pipiens (6-7 d old) were reared (30 per sample) in $60 \mathrm{ml}$ of distilled water in the presence of SCPI-1 $(0.54-34.8 \mu \mathrm{M})$ and fed fish food (TetraMin). Control larvae were reared in correspondingly diluted DMSO (solvent for stock solutions of each compound). Mortality in the control larvae was $<10 \%$. The number of dead larvae was counted $72 \mathrm{~h}$ after the SCPIs were applied. The $\mathrm{LC}_{50}$ value was determine by converting the percentage of mortality to the Probit unit, and then plotting the percentage of mortality (Probit unit) on the y-axis and the log [concentration] on the x-axis.

Starting from the first instar, Ae. aegypti larvae (50-60 larvae per sample) were reared in 60 $\mathrm{ml}$ of distilled water in the presence of an SCPI-1 (1.56-25 $\mu \mathrm{M})$ and fed fish food (TetraMin). Control larvae were reared in correspondingly diluted DMSO (solvent for stock solutions of each compound). Mortality in the control larvae was $<10 \%$. At $72 \mathrm{~h}$ and $6 \mathrm{~d}$, dead larvae and pupae were recorded. After $12 \mathrm{~d},>95 \%$ adults eclosed in the controls. The $\mathrm{LC}_{50}$ value at $72 \mathrm{~h}$ and $12 \mathrm{~d}$ was determine by converting the percentage of mortality to the Probit unit, and then plotting the percentage of mortality (Probit unit) on the y-axis and the log [concentration] on the $\mathrm{x}$-axis.

Larvae collected from wild populations of $C x$. restuans were mostly between second to third instar. Larvae (50 - 60 larvae per sample) were reared in $60 \mathrm{ml}$ of distilled water in the presence of SCPI-1 (0.78-25 $\mu \mathrm{M})$ and fed fish food (TetraMin). Control larvae were reared in correspondingly diluted DMSO (solvent for stock solutions of each compound). Mortality in the control larvae was $<10 \%$. Dead larvae and pupae were recorded after $72 \mathrm{~h}$. The $\mathrm{LC}_{50}$ value at $72 \mathrm{~h}$ was determined by converting the percentage of mortality to the Probit unit, and then plotting the percentage of mortality (Probit unit) on the y-axis and the log [concentration] on the $\mathrm{x}$-axis.

Pharate Ae. aegypti fourth instars were staged by physical appearance: the visible dark black hairs of fourth stadium larva wrapped around the body under the thorax and abdominal cuticle of the third instar (Christophers, 1960). Larvae selected by this criteria ecdyse during a 1-h period. Pharate fourth instars were selected and transferred into a new container, and newly molted fourth instars were collected. The newly molted fourth instars were place in $60 \mathrm{ml}$ of distilled water in the presence of an SCPI-1 (0.78-25 $\mu \mathrm{M})$ and fed fish food (TetraMin). At 72 $\mathrm{h}$ and $6 \mathrm{~d}$, dead larvae and pupae were recorded. After $6 \mathrm{~d},>90 \%$ adults had eclosed in the controls. Mortality in the control larvae was $<10 \%$. The $\mathrm{LC}_{50}$ value at $72 \mathrm{~h}$ and $6 \mathrm{~d}$ was determined by converting the percentage of mortality to the Probit unit, and then plotting the percentage of mortality (Probit unit) on the $y$-axis and the log [concentration] on the x-axis. 


\section{Tryptophan Fluorescent Quenching Assays}

AeSCP-2 has only one tryptophan residue that is situated within the hydrophobic ligand binding cavity, although it does not directly interact with the ligand (Dyer et al. 2003). To verify whether the interaction between SCPI-1 and AeSCP-2 causes protein structural changes, we measured the tryptophan fluorescent emission with and without SCPI-1. Recombinant AeSCP-2 (1 mg/ $\mathrm{ml})$ was incubated at room temperature with and without SCPI-1 $(1 \mu \mathrm{M})$, and the controls were guanidine (4.5 M) denatured AeSCP-2 with and without SCPI-1. Fluorescent emission of tryptophan in AeSCP-2 was scanned using a fluorescence microplate reader (Sepctra MAX Gemini XS, Molecular Devices, Sunnyvale, CA).

\section{Effects of SCPIs on Cholesterol Uptake in Fourth Instars}

To test whether SCPIs affect cholesterol uptake in vivo, ten 24-h-old fourth instars that had been treated with $6.25 \mu \mathrm{M} \mathrm{SCPI}-1$ or SCPI-2 since the first stadium were put in $5 \mathrm{ml}$ of doubledistilled waster containing $\left[{ }^{3} \mathrm{H}\right]$ cholesterol $(0.2 \mu \mathrm{Ci} / \mathrm{ml}), 6.25 \mu \mathrm{M}$ SCPI-1, or SCPI-2 (diluted from a $100 \mathrm{mM}$ stock solution in DMSO) along with fish food (TetraMin). Control larvae were selected from the same batch of larvae and treated with correspondingly diluted DMSO (solvent for SCPIs). After the 24-h labeling, the larvae were washed with distilled water and 70\% ethanol to remove $\left[{ }^{3} \mathrm{H}\right]$ cholesterol from the exterior of the body. Total cholesterol from groups of two larvae was extracted from the whole body as described previously (Blitzer et al. 2005); incorporated $\left[{ }^{3} \mathrm{H}\right]$ cholesterol was determined and presented as $\mathrm{dpm} / \mathrm{mg}$ total soluble protein.

\section{Statistics}

Student $t$-test was used in cases that a pair of treatments was compared with determine the significance of the differences.

\section{Results and Discussion \\ Toxicity of SCPI-1 in Larvae of Mosquito Species}

SCPIs are toxic to Ae. aegypti larvae (Kim et al. 2005). Whether other mosquito species are susceptible to SCPIs was unknown. We speculated that An. gambiae and Cx. pipiens larvae would be susceptible to SCPI-1 because An. gambiae has a predicted homologous protein (ENSANGP00000020493) of AeSCP-2 with 85\% identify (Lan and Wessely 2004), and $C x$. pipiens quinquefasciatus SCP-2 (AY196002) has 86\% identity to AeSCP-2 (Fig. 1). To investigate whether SCPI-1 is lethal to other mosquito species, SCPI-1 treatment was applied to third instars from laboratory strains of An. gambiae and Cx. pipiens pipiens.

The average $\mathrm{LC}_{50}$ values of SCPI-1 in An. gambiae and Cx. pipiens pipiens were 14.1 and 5.2 $\mu \mathrm{M}$, respectively (Table 1). The susceptibility of An. gambiae and Cx. pipiens to SCPI-1 was consistent with the fact that both An. gambiae and Cx. pipiens have AeSCP-2 homologous genes with high identities. The reported 72-h $\mathrm{LC}_{50}$ for third instars of An. gambiae and $C x$. pipiens to SCPI-1 was between the $\mathrm{LC}_{50}$ values reported for first and fourth instars of $A e$. aegypti larvae (5.2 and $38.7 \mu \mathrm{M}$, respectively), which indicates SCPI-1 may have a broader toxicity spectrum in mosquito species.

To examine whether SCPIs are lethal to mosquito larvae from the wild populations, SCPI-1 was tested using larvae collected during the summer around Madison, WI (see Materials and Methods). Mosquito larvae samples were brought back to the laboratory, 20 - 60 larvae were randomly selected from the sample, and species of the larvae were identified using keys described by Siverly (1972). Samples that had $>90 \%$ of the larvae from a single mosquito species were used in toxicity assays. $C x$. restuans dominant in samples collected; therefore, SCPI-1 toxicity assays were only performed in larvae from wild populations of $C x$. restuans. The $\mathrm{LC}_{50}$ value of SCPI-1 in Cx. restuans was $14.9 \mu \mathrm{M}$ (Table 1). The results showed that 
larvae from wild populations of $C x$. restuans were susceptible to SCPI-1, and the $\mathrm{LC}_{50}$ was comparable with that of the laboratory-tested species: Ae. aegypti, An. gambiae, and $C x$. pipiens (Table 1). When treatments started at the same stage, the $\mathrm{LC}_{50}$ values of SCPI-1 for different mosquito species varied by more than two-fold (Table 1, An. gambiae and $C x$. pipiens). The slopes calculated from the concentration-dependent lethality of SCPI-1 for An. gambiae and $C x$. pipiens were 0.338 and 0.433 , respectively (Fig. 2), indicating $C x$. pipiens was more sensitive to SCPI-1 than An. gambiae. The varied susceptibilities of difference species to SCPI-1 might be due to two reasons. First, the growth rate of the two mosquito species is slightly different, which may result in different susceptibility to a growth regulator such as SCPI-1. Second, the protein structures of the mosquito SCP-2s from these species may be slightly different, rendering different affinities to SCPI-1 and leading to different sensitivities to SCPI-1 treatment. Further investigations in the SCP-2 proteins from other mosquito species will clarify the varied susceptibilities to SCPI-1.

The developmental stages when treatment started were different among Ae. aegypti and the other mosquito species tested, which may play a role in susceptibility to SCPI-1. The bioassays in laboratory strains of An. gambiae and Cx. pipiens were conducted in the third instar, whereas the toxicity assays in Ae. aegytpi were performed in first and fourth instars. Because SCPIs inhibit the absorption of an essential nutrient, it is highly possible that larvae in late stages are less susceptible to SCPIs than those in early stages. Staged Ae. aegypti larvae were used to measure the $\mathrm{LC}_{50}$ values of SCPI-1 in first and fourth instars. When SCPI-1 treatment started at the beginning of fourth stadium, the $72-\mathrm{h} \mathrm{LC}_{50}$ values increase to $38.7 \mu \mathrm{M}$ (Table 1), indicating older larvae were less susceptible to SCPI treatment. The result is consistent with the nature of a growth inhibitory larvicide. Therefore, the higher $\mathrm{LC}_{50}$ obtained in fourth instars of Ae. aegypti (Table 1) were most likely due to the treatment being started at a later stage of larval development.

The mortality rates in SCPI-1-treated larvae did not change significantly $3 \mathrm{~d}$ after the treatments. When treatments started at the first instar, the $\mathrm{LC}_{50}$ values at 3 and $12 \mathrm{~d}$ (adult emerge) were 5.05 and 4.04 , respectively (Table 2 ). When treatments started at the fourth instar, the $\mathrm{LC}_{50}$ values at 3 and $6 \mathrm{~d}$ (adult emerge) were 38.7 and 24.04, respectively (Table 2). The results indicate that most of the lethal effects of SCPI- 1 take place within the first $3 \mathrm{~d}$ of treatment, regardless the developmental stages of the larvae. The results suggest that SCPI-1 may have low residual activity, because the application of SCPI-1 only occurred once at the beginning of the treatment. The residual activity of SCPI-1 needs further testing.

\section{Tryptophan Fluorescent Quenching in AeSCP-2 Induced by SCPI-1}

SCPI-1 was identified through high-throughput screening of a chemical library by using AeSCP-2 as the target protein (Kim et al. 2005). SCPI-1 competes with NBD-cholesterol, a cholesterol analog, for AeSCP-2 binding in a dose-response manner, although the precise mechanism is unknown (Kim et al. 2005). Whether, SCPI-1 causes conformational changes in AeSCP-2 is unclear. Fluorescence measurements from intrinsic sources are often useful for reporting conformational states of proteins (Lakowicz 1999). Fluorescence emission from tryptophan (Trp) residues in a protein is extremely sensitive to perturbations in the local environment, mostly due to different degrees of quenching. For example, Trp buried in hydrophobic domain of native protein has maximal fluorescent intensity usually at 330-333 $\mathrm{nm}$, whereas in denatured protein Trp fluorescent emission shows a red shift to $350-353 \mathrm{~nm}$ (King 1994, Reshetnyak and Burstein 2001). AeSCP-2 has a single Trp residue at amino acid position $44\left(\mathrm{~W}^{44}\right)$ (Krebs and Lan 2003). In the native state, AeSCP-2 fluorescent emission peaks at $333 \mathrm{~nm}$ (Fig. 3, blue line), indicating $\mathrm{W}^{44}$ is not exposed to the surface of the protein (King 1994). This result is consistent with the $\mathrm{x}$-ray protein crystal structure that shows $\mathrm{W}^{44}$ in AeSCP-2 is situated within the hydrophobic ligand binding cavity, although it does not 
directly interact with the ligand (Dyer et al. 2003). In guanidine denatured AeSCP-2, the peak of fluorescent emission was at $355 \mathrm{~nm}$ (Fig. 3, pink line), which is consistent with a "red shift" of Trp fluorescence in denatured proteins.

The fluorescence emission spectrums of denatured AeSCP-2 overlay each other with and without SCPI-1 (Fig. 1, pink and purple lines), which indicates that SCPI-1 did not quench Trp fluorescence in the denatured AeSCP-2. With the addition of SCPI-1, there was a major spectral shift for AeSCP-2 compared with native AeSCP-2 without inhibitor (Fig. 3, red line). SCPI-1 seems to quench the tryptophan's fluorescent emission in native AeSCP-2 protein (Fig. 3, blue and red lines), either through direct fluorescent resonance energy transfer or by changing the solvent exposure of buried Trp in AeSCP-2. The result indicates that SCPI-1 directly interacts with native AeSCP-2, which leads to changes in tryptophan residue's fluorescence emission. The results of Trp fluorescent quenching by SCPI-1 in native AeSCP-2 provided evidence for the first time that SCPI-1 directly interacts with AeSCP-2 and causes conformational change in AeSCP-2.

\section{Effect of SCPIs on Cholesterol Uptake in Mosquito Larvae}

SCPI-1 and SCPI-2 have been shown to have larvicidal activities in Ae. aegypti larvae (Kim et al. 2005). To investigate whether SCPI inhibits cholesterol uptake in larvae, larvae were treated with $6.25 \mu \mathrm{M} \mathrm{SCPI}-1$ or SCPI-2 starting at the first instar, and 24-h-old fourth instars were transferred into in water containing $\left[{ }^{3} \mathrm{H}\right]$ cholesterol in the presence of $6.25 \mu \mathrm{M}$ SCPI-1 or SCPI- 2 for $24 \mathrm{~h}$. At the end of the labeling period, the larvae were 48 -h post-third molt, at which point the midgut has undergone metamorphic changes and has lost most of its neutral lipids content (Parthasarathy and Palli 2006, Nishiura et al. 2007). $\left[{ }^{3} \mathrm{H}\right]$ Cholesterol from the exterior of the body was washed off using $70 \%$ ethanol and labeled cholesterol absorbed by larvae during a 24 -h period was measured. SCPI-treated fourth instars experienced a $30 \%$ decrease $(P<0.04)$ in $\left[{ }^{3} \mathrm{H}\right]$ cholesterol uptake within $24 \mathrm{~h}$, compared with the controls (Fig. 4, hatched and black bars). The result was consistent with the $33 \%$ decrease of cholesterol accumulation in AeSCP-2 RNA interference treatment fourth instars (Blitzer et al. 2005). Therefore, one of the modes of SCPI action in vivo seems to be the inhibitory effect on the SCP-2 mediated cholesterol absorption pathway.

In conclusion, the results from this study provided evidence that SCPI-1 directly interacts with AeSCP-2, which inhibits ligand binding in AeSCP-2. Results from bioassays showed that SCPI-1 has a broad toxicity in mosquito species, indicating these mosquitoes may have homologous SCP-2 with similar function.

\section{Acknowledgements}

We thank Drs. Bruce Christensen and Susan Paskewitz for the supply of mosquito eggs. We thank David H. Dyer for technical support. This work was supported by the University of Wisconsin-Madison College of Agriculture and Life Sciences' USDA-CSREES Hatch project WIS04963, by National Institutes of Health grant 5R01 AI-067422, and by grant W9113M-05-1-0006 from the Deployed War Fighter Protection Research Program administered by the U.S. Armed Forces Pest Management Board (to Q.L.).

\section{References Cited}

Arrese EL, Canavoso LE, Jouni ZE, Pennington JE, Tsuchida K, Wells MA. Lipid storage and mobilization in insects: current status and future directions. Insect Biochem Mol Biol 2001;31:7-17. [PubMed: 11102830]

Blitzer EJ, Vyazunova I, Lan Q. Functional analysis of AeSCP-2 using gene expression knockdown in the yellow fever mosquito, Aedes aegypti. Insect Mol Biol 2005;14:301-307. [PubMed: 15926899]

[CDC] Centers for Disease Control and Prevention. Centers for Disease Control and Prevention homepage. 2003. http://www.cdc.gov/) 
Chino H, Kitazawa K. Diacylglycerol-carrying lipoprotein of hemolymph of the locust and some insects. J Lipid Res 1981;22:1042-1052. [PubMed: 6795289]

Christophers, SR. Aedes aegypti (L). The yellow fever mosquito. Its life history, bionomics and structure. Cambridge University Press; Cambridge, United Kingdom: 1960.

Dyer DH, Lovell S, Thoden JB, Holden HM, Rayment I, Lan Q. The structural determination of an insect sterol carrier protein- 2 with a ligand bound $\mathrm{C} 16$ fatty acid at $1.35 \mathrm{~A}$ resolution. $\mathrm{J}$ Biol Chem 2003;278:39085-39091. [PubMed: 12855689]

Focks DA, Brenner RJ, Hayes J, Daniels E. Transmission thresholds for dengue in terms of Aedes aegypti pupae per person with discussion of their utility in source reduction efforts. Am J Trop Med Hyg 2000;62:11-18. [PubMed: 10761719]

Gallegos AM, Atshaves BP, Storey SM, Starodub O, Petrescu AD, Huang H, McIntosh AL, Martin GG, Chao H, Kier AB, et al. Gene structure, intracellular localization, and functional roles of sterol carrier protein-2. Prog Lipid Res 2001;40:498-563. [PubMed: 11591437]

Halstead SB. The XXth century dengue pandemic: need for surveillance and research. World Health Stat Q 1992;45:292-298. [PubMed: 1462664]

Kim MS, Wessely V, Lan Q. Identification of mosquito sterol carrier protein-2 inhibitors. J Lipid Res 2005;46:650-657. [PubMed: 15627652]

King L. Effects of denaturant and pressure on the intrinsic fluorescence of titin. Arch Biochem Biophys 1994;311:251-257. [PubMed: 8203887]

Krebs KC, Lan Q. Isolation and expression of a sterol carrier protein-2 gene from the yellow fever mosquito, Aedes aegypti. Insect Mol Biol 2003;12:51-60. [PubMed: 12542635]

Lakowicz, J. Principles of fluorescence spectroscopy. Vol. 2nd. Plenum Press; New York: 1999.

Lan Q, Massey RJ. Subcellular localization of mosquito sterol carrier protein-2 and sterol carrier proteinx. J Lipid Res 2004;45:1468-1474. [PubMed: 15145982]

Lan Q, Wessely V. Expression of a sterol carrier protein-x gene in the yellow fever mosquito, Aedes aegypti. Insect Mol Biol 2004;13:519-529. [PubMed: 15373808]

Moller G, Luders J, Markus M, Husen B, Van Veldhoven PP, Adamski J. Peroxisome targeting of porcine 17beta-hydroxysteroid dehydrogenase type IV/D-specific multifunctional protein 2 is mediated by its C-terminal tripeptide AKI. J Cell Biochem 1999;73:70-78. [PubMed: 10088725]

Mourya DT, Hemingway J, Leake CJ. Changes in enzyme titres with age in four geographical strains of Aedes aegypti and their association with insecticide resistance. Med Vet Entomol 1993;7:11-16. [PubMed: 8435483]

Mourya DT, Gokhale MD, Mishra AC. Biochemical basis of DDT-resistance in Aedes aegypti population from a dengue affected area in Shahjahanpur city. Indian J Med Res 1994;99:212-215. [PubMed: 7927578]

Nishiura JT, Burgos C, Aya S, Goryacheva Y, Lo W. Modulation of larval nutrition affects midgut neutral lipid storage and temporal pattern of transcription factor expression during mosquito metamorphosis. J Insect Physiol 2007;53:47-58. [PubMed: 17123540]

Parthasarathy R, Palli SR. Stage- and cell-specific expression of ecdysone receptors and ecdysoneinduced transcription factors during midgut remodeling in the yellow fever mosquito, Aedes aegypti. J Insect Physiol. 2006

Rawlins SC. Spatial distribution of insecticide resistance in Caribbean populations of Aedes aegypti and its significance. Rev Panam Salud Publica 1998;4:243-251. [PubMed: 9924507]

Rawlins SC, Wan JO. Resistance in some Caribbean populations of Aedes aegypti to several insecticides. J Am Mosq Control Assoc 1995;11:59-65. [PubMed: 7542312]

Renshaw PF, Janoff AS, Miller KW. On the nature of dilute aqueous cholesterol suspensions. J Lipid Res 1983;24:47-51. [PubMed: 6833881]

Reshetnyak YK, Burstein EA. Decomposition of protein tryptophan fluorescence spectra into log-normal components. II. The statistical proof of discreteness of tryptophan classes in proteins. Biophys J 2001;81:1710-1734. [PubMed: 11509383]

Schroeder F, Gallegos AM, Atshaves BP, Storey SM, McIntosh AL, Petrescu AD, Huang H, Starodub $\mathrm{O}$, Chao H, Yang H, et al. Recent advances in membrane microdomains: rafts, caveolae, and intracellular cholesterol trafficking. Exp Biol Med (Maywood) 2001;226:873-890. [PubMed: 11682693] 
Siverly, RE. Mosquitoes of Indiana. Indiana State Board of Health; Indianapolis, IN: 1972.

Thompson JD, Higgins DG, Gibson TJ. Nucleic Acids Res 1994;22:4673-4680. [PubMed: 7984417]

Vyazunova I, Wessely V, Kim MS, Lan Q. Identification of two sterol carrier protein-like genes in the yellow fever mosquito, Aedes aegypti. Insect Mol Biol. 2007in press

Yancey PG, Rodrigueza WV, Kilsdonk EP, Stoudt GW, Johnson WJ, Phillips MC, Rothblat GH. Cellular cholesterol efflux mediated by cyclodextrins. Demonstration of kinetic pools and mechanism of efflux. J Biol Chem 1996;271:16026-16034. [PubMed: 8663188]

Zdobnov EM, von Mering C, Letunic I, Torrents D, Suyama M, Copley RR, Christophides GK, Thomasova D, Holt RA, Subramanian GM, et al. Comparative genome and proteome analysis of Anopheles gambiae and Drosophila melanogaster. Science (Wash, DC) 2002;298:149-159. 
AesCP-2
CxSCP-2
AnSCP-2

AeSCP-2

$\mathrm{CxSCP}-2$

AnSCP-2
MSLKSDEVFAKIAKRLESIDPANRQVEHVYKFRITQGGKVVKNWVMDLKNVKLVESDDAA MSLKSNEVFAKIAKRLENIDPANRQVQQVYKERITKDGKVVKNWVMDLKAVKLTESDGDA MALKSDPVFERIAKRLESIDPNNRQVQQVYKFRIQQNGTVVKTWVLDLKAVKLTEGDGPA

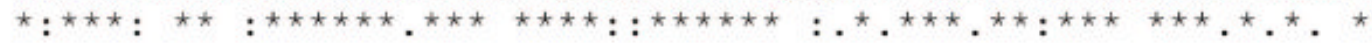
EATLTMEDDIMFAIGTGALPAKEAMAQDKMEVDGQVELIFLLEPFIASLKEATLIMDDE IMFAIGTGAMPAKEAMAQDKMEVRGQVELIFLLEPFIASLKEATLTMEDDIMFALGTGAMPAKEALAQDKLDVEGQVELIFLLEPFIASLKK



Fig. 1.

Amino acid alignment of mosquito SCP-2 proteins. Ae aegypti SCP-2 (AeSCP-2:

AAO34708). Identical residue at a given position is marked by a star under the sequence. Conservative substitution of residue at a given position is marked by a dot or double dots under the sequence. The alignment was prepared with the program CLUSTALW version 1.8 (Thompson et al. 1994). An. gambiae SCP-2 (AnSCP-2: ENSANGP00000020493). Cx. pipiens quinquefasciatus (CxSCP-2: AY196002). Red residues directly coordinate the carboxyl group of palmatic acid (Dyer et al. 2003). Yellow residues directly interact with the acyl chain of palmatic acid (Dyer et al. 2003). 


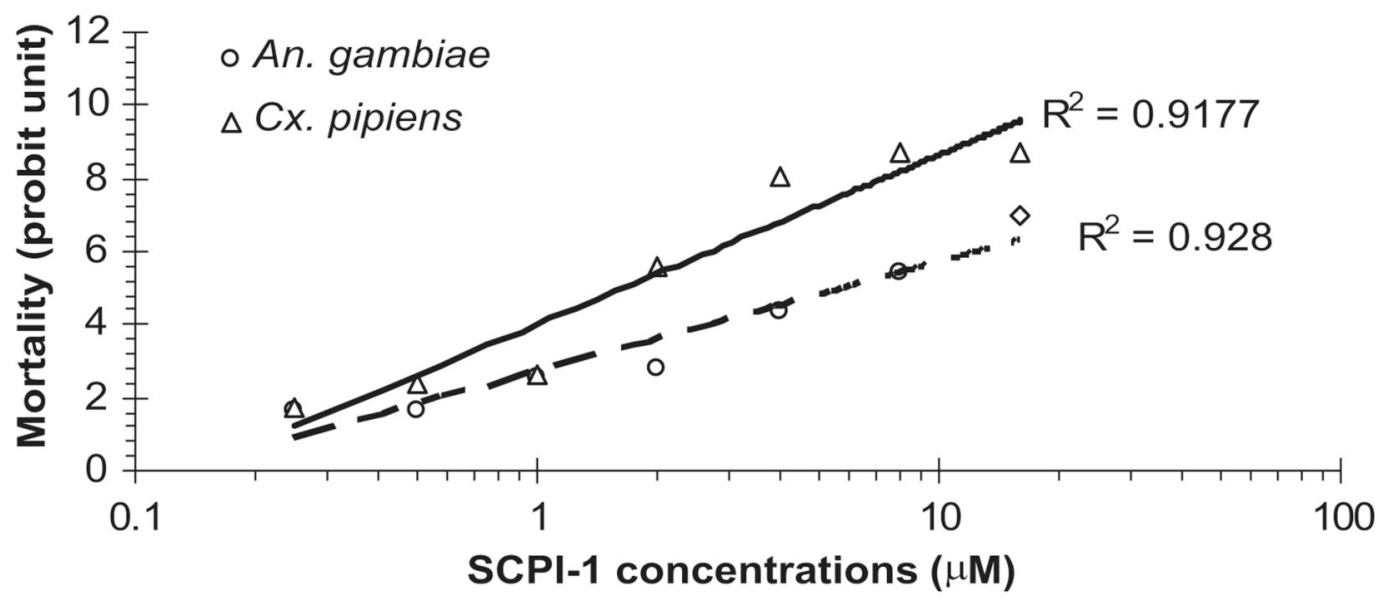

Fig. 2.

Dose-response lethal effects of SCPI-1. Data represent the average of the replicates (see Table 1) at each treated concentration starting at the third instar of An. gambiae and Cx. pipiens pipiens. The mortality is transformed to Probit units. 


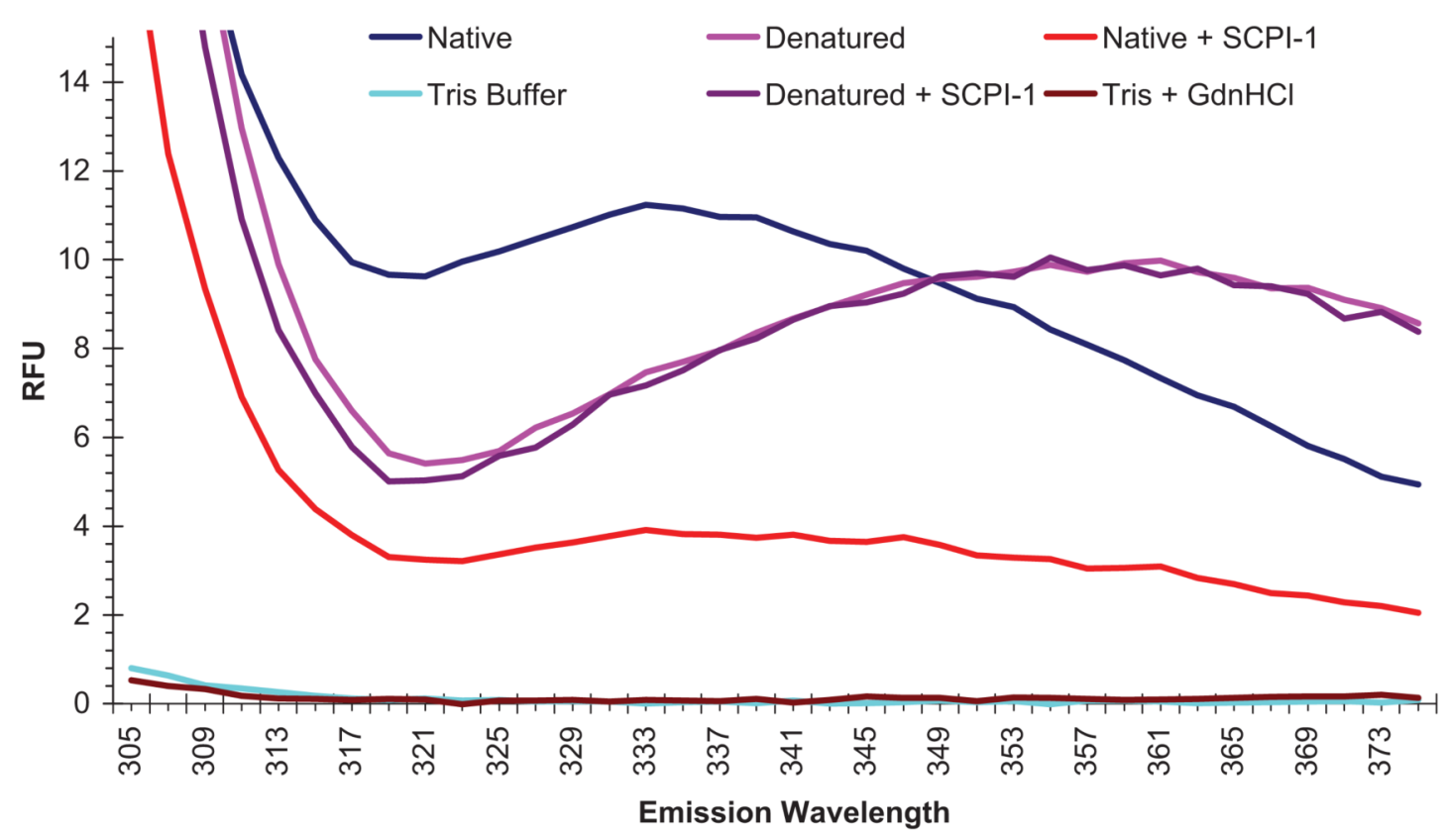

Fig. 3.

Fluorescent emission scan of AeSCP-2. GdnHCl, guanidine hydrochloride solution for denaturing AeSCP-2. Tris, 50 mM Tris buffer, pH 7.4, in which AeSCP-2 was stored. RFU, relative fluorescent unit. The data were the average readings of triplicates for each data point. 


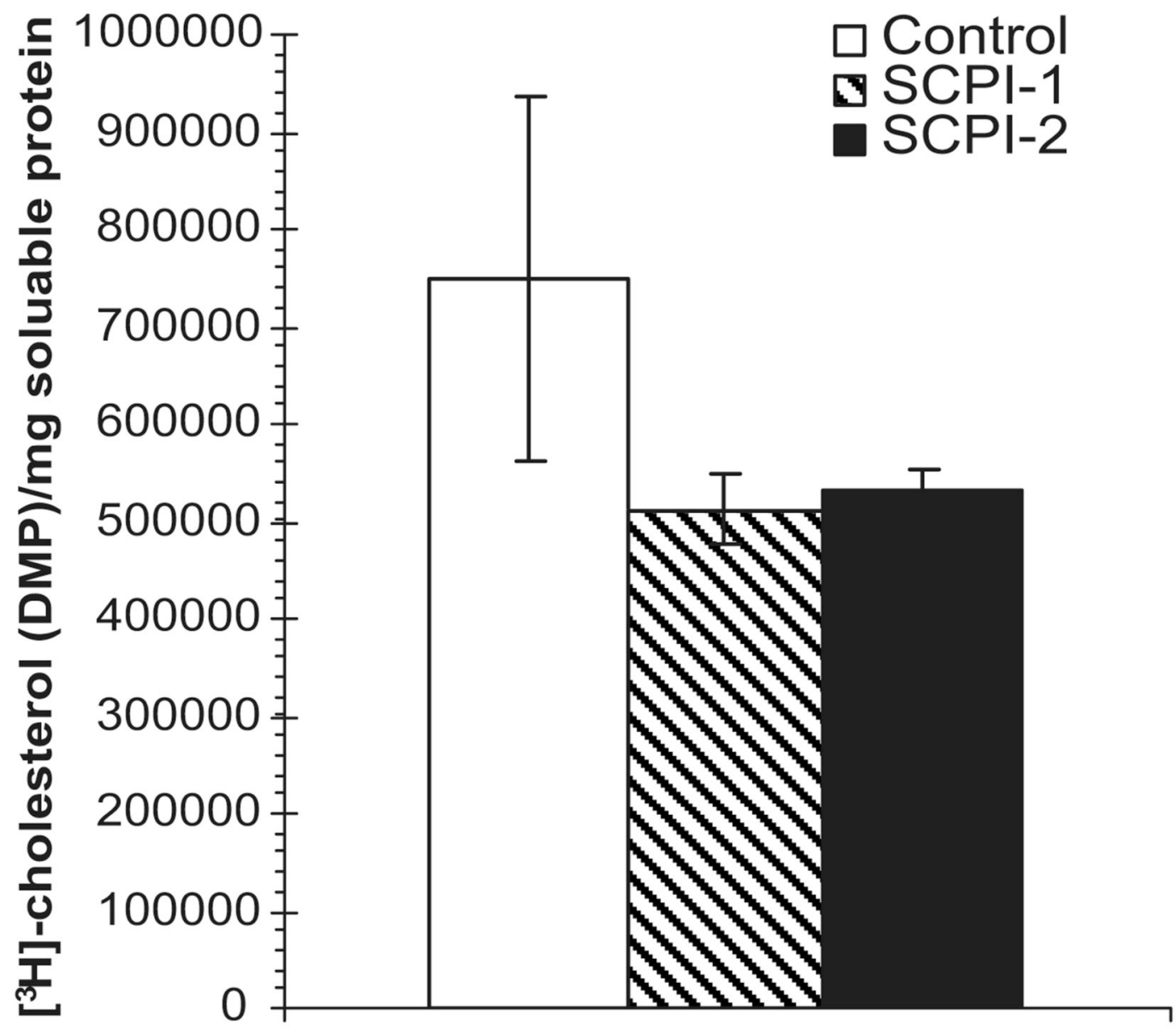

Fig. 4.

Effect of SCPI treatment on accumulation of $\left[{ }^{3} \mathrm{H}\right]$ cholesterol in fourth instars. SCPI-treated fourth instars were labeled with $\left[{ }^{3} \mathrm{H}\right]$ cholesterol for $24 \mathrm{~h}$, and the amount of absorbed $\left[{ }^{3} \mathrm{H}\right]$ cholesterol was measured (see Materials and Methods). White bars, controls; hatched bars, SCPI-1 treated; and black bars, SCPI-2 treated. Data are mean \pm SD $(n=5)$. 
Table 1

Third-day larvicidal activities of SCPI-1 in mosquitoes

\begin{tabular}{lc}
\hline Species & LC $_{\mathbf{5 0}} \pm \mathbf{9 5 \%} \mathbf{C I}$ \\
\hline Ae. aegypti (first instar, $n=7$ ) & $5.2 \pm 0.7$ \\
An. gambiae (third instar, $n=3$ ) & $14.1 \pm 6.5$ \\
Cx. pipiens pipiens (third instar, $n=4)$ & $5.2 \pm 1.7$ \\
Cx. restuans (second-third instar, $n=4)$ & $14.9 \pm 2.9$ \\
Ae. aegypti (fourth instar, $n=7$ ) & $38.7 \pm 3.4$ \\
\hline
\end{tabular}


Table 2

SCPI-1 larvicidal activities ( $\mathrm{LC}_{50} \pm 95 \%$ CI.) in Ae. aegypti larvae (all $\left.n=7\right)$ at different durations

\begin{tabular}{lcc}
\hline Starting treatment & 3 d after treatment & At adult emergence \\
\hline First instar & $5.2 \pm 0.7$ & $4.04 \pm 0.4$ \\
Fourth instar & $38.7 \pm 3.4$ & $24.4 \pm 2.9$ \\
\hline
\end{tabular}

